\title{
Oncological management and obstetric and neonatal outcomes for women diagnosed with cancer during pregnancy: a 20-year international cohort study of 1170 patients
}

\author{
Jorine de Haan*, Magali Verheecke*, Kristel Van Calsteren, Ben Van Calster, Roman G Shmakov, Mina Mhallem Gziri, Michael J Halaska, \\ Robert Fruscio, Christianne A R Lok, Ingrid A Boere, Paolo Zola, Petronella B Ottevanger, Christianne J M de Groot, Fedro A Peccatori, \\ Karina Dahl Steffensen, Elyce H Cardonick, Evgeniya Polushkina, Lukas Rob, Lorenzo Ceppi, Gennady T Sukhikh, Sileny N Han, Frédéric Amant, \\ for the International Network on Cancer, Infertility and Pregnancy (INCIP)
}

\section{Summary}

Background Awareness is growing that cancer can be treated during pregnancy, but the effect of this change on maternal and neonatal outcomes is unknown. The International Network on Cancer, Infertility and Pregnancy (INCIP) registers the incidence and maternal, obstetric, oncological, and neonatal outcomes of cancer occurring during pregnancy. We aimed to describe the oncological management and obstetric and neonatal outcomes of patients registered in INCIP and treated in the past 20 years, and assess associations between cancer type or treatment modality and obstetric and neonatal outcomes.

Methods This descriptive cohort study included pregnant patients with cancer registered from all 37 centres (from 16 countries) participating in the INCIP registry. Oncological, obstetric, and neonatal outcome data of consecutive patients diagnosed with primary invasive cancer during pregnancy between Jan 1, 1996, and Nov 1, 2016, were retrospectively and prospectively collected. We analysed changes over time in categorical patient characteristics outcomes, and treatment methods with log-binomial regression. We used multiple logistic regression to analyse preterm, prelabour rupture of membranes (PPROM) or preterm contractions, small for gestational age, and admission to the neonatal intensive care unit (NICU). The INCIP registry study is registered with ClinicalTrials.gov, number NCT00330447, and is ongoing.

Findings 1170 patients were included in the analysis and 779 (67\%) received treatment during pregnancy. Breast cancer was the most common malignant disease (462 [39\%]). Every 5 years, the likelihood of receiving treatment during pregnancy increased (relative risk [RR] 1.10, 95\% CI 1.05-1.15), mainly related to an increase of chemotherapeutic treatment $(1 \cdot 31,1 \cdot 20-1 \cdot 43)$. Overall, $955(88 \%)$ of 1089 singleton pregnancies ended in a livebirth, of which 430 (48\%) of 887 pregnancies ended preterm. Each 5 years, we observed more livebirths (RR 1.04, 95\% CI 1.01-1.06) and fewer iatrogenic preterm deliveries $(0.91,0 \cdot 84-0 \cdot 98)$. Our data suggest a relationship between platinum-based chemotherapy and small for gestational age (odds ratio [OR] 3·12, 95\% CI 1.45-6.70), and between taxane chemotherapy and NICU admission (OR 2.37, 95\% CI 1·31-4.28). NICU admission seemed to depend on cancer type, with gastrointestinal cancers having highest risk (OR 7.13, 95\% CI 2.86-17.7) and thyroid cancers having lowest risk $(0 \cdot 14,0.02-0.90)$ when compared with breast cancer. Unexpectedly, the data suggested that abdominal or cervical surgery was associated with a reduced likelihood of NICU admission (OR $0.30,95 \% \mathrm{CI}$ $0 \cdot 17-0 \cdot 55)$. Other associations between treatment or cancer type and outcomes were less clear.

Interpretation Over the years, the proportion of patients with cancer during pregnancy who received antenatal treatment increased, especially treatment with chemotherapy. Our data indicate that babies exposed to antenatal chemotherapy might be more likely to develop complications, specifically small for gestational age and NICU admission, than babies not exposed. We therefore recommend involving hospitals with obstetric high-care units in the management of these patients.

Funding Research Foundation-Flanders, European Research Council, Charles University, Ministry of Health of the Czech Republic.

\section{Introduction}

The incidence of cancer during pregnancy is estimated to be one in 1000 pregnancies..$^{1-3}$ Breast cancer, haematological cancer, cervical cancer, and melanoma are the most commonly diagnosed malignant diseases during pregnancy.,5 Although cohort studies of maternal and fetal outcomes in these women have included overall maternal and obstetric outcomes, 4,5 most of these studies have small sample sizes and short follow-ups.
Lancet Oncology 2018 Published Online January 24, 2018 http://dx.doi.org/10.1016/ S1470-2045(18)30059-7 See Online/Comment http://dx.doi.org/10.1016/ S1470-2045(18)30058-5

*Contributed equally

Department of Oncology (J de Haan MD, M Verheecke MD SN Han PhD, Prof F Amant PhD) and Department of Development and Regeneration

(K Van Calsteren PhD, $B$ Van Calster PhD), KU Leuven, Leuven, Belgium; Department of Obstetrics and Gynaecology, VU University Medical Center, Amsterdam, Netherlands (J de Haan,

Prof (J M de Groot PhD); Division of Gynaecologic Oncology (M Verheecke, SN Han, Prof F Amant) and Department of Obstetrics and Gynaecology (K Van Calsteren), University Hospitals Leuven, Leuven, Belgium; Federal State Budget Institution "Research Center for Obstetrics, Gynecology and Perinatology", Ministry of Healthcare of the Russian Federation, Moscow, Russia (R G Shmakov PhD, E Polushkina PhD, Prof GT Sukhikh PhD); Department of Obstetrics, Cliniques Universitaires St Luc, UCL, Sint-Lambrechts-Woluwe, Belgium (M Mhallem Gziri PhD); Faculty Hospital Kralovske Vinohrady and 3rd Medical Faculty, Charles University, Prague, Czech Republic (M) Halaska PhD, Prof $L$ Rob PhD); Clinic of Obstetrics and Gynecology, University of Milan Bicocca San Gerardo Hospital, Monza, Italy (R Fruscio PhD, LCeppi MD); 
Center for Gynaecologic Oncology Amsterdam, Antoni van LeeuwenhoekNetherlands Cancer Institute, Amsterdam, Netherlands (C A R Lok PhD, Prof F Amant); Department of Medical Oncology, Erasmus MC Cancer Institute, Erasmus University Medical Center, Rotterdam, Netherlands (I A Boere PhD); Department of Surgical Sciences, University of Turin, Turin, Italy ( $\mathrm{P}$ Zola PhD); Department of Medical Oncology, Radboud University Nijmegen Medical Center, Nijmegen, Netherlands (P B Ottevanger PhD); Division of Gynaecologic Oncology, European Institute of Oncology, Milan, Italy (FA Peccatori PhD); Department of Oncology, Vejle Hospital, Vejle, Denmark (K Dahl Steffensen PhD); Institute of Regional Health Research, Faculty of Health Sciences, University of Southern Denmark, Odense, Denmark (K Dahl Steffensen); Department of Obstetrics and Gynaecology, Cooper University Health Care, Camden, NJ, USA

(Prof E H Cardonick PhD); and Centre for Gynaecologic Oncology Amsterdam, Academical Medical Center, Amsterdam, Netherlands (Prof F Amant)

Correspondence to: Dr Frédéric Amant, Center for Gynaecologic Oncology Amsterdam, Antoni van Leeuwenhoek-Netherlands Cancer Institute, Amsterdam, Netherlands f.amant@nki.nl

See Online for appendix

For the INCIP registry see http:// www.cancerinpregnancy.org
Research in context

\section{Evidence before this study}

We searched PubMed on March 30, 2017, for articles on cohorts of patients with cancer during pregnancy that described obstetric and oncological outcomes, published between Jan 1, 1996, and Dec 31, 2016, using the following keywords: "pregnancy", "cancer", "tumour", "neoplasm", "pregnancy outcome", and "neonatal outcome". The search was restricted to publications in English. We reviewed references from appropriate articles to identify additional studies, resulting in many articles on cancer before or after pregnancy but not specifically during pregnancy. After selection by abstract and full text, we included 71 studies with sample sizes ranging from nine to 984 patients. A cohort was considered large and included if it contained 50 or more patients, resulting in 25 articles describing either obstetric or neonatal outcome. No articles on management changes were published. 23 studies reported risk or frequency of prematurity; 12 found an increased frequency or risk of prematurity in cancer in pregnancy and four studies did not find an increase. Neonatal outcomes were reported to some extent in all studies. One study showed an increased frequency or risk of neonatal mortality, whereas ten did not find such an increase. Increased risk of admission to the neonatal intensive care unit (NICU) was found in one study, whereas three found no such increase. Overall, small for gestational age was assessed in 22 articles and was increased in five studies, whereas 13 studies did not find an overall increased risk. No studies found an increased frequency or risk of congenital anomalies.

In 2010, our group published the first epidemiological data on cancer during pregnancy, from the registry of the International Network on Cancer, Infertility and Pregnancy (INCIP). ${ }^{4}$ Subsequently, we ${ }^{6,7}$ reported findings from two prospective follow-up studies of children with antenatal chemotherapy exposure; we found no clinical difference in neurocognitive or cardiac development between the treatment and the control group. In both groups, preterm delivery was the main risk factor for paediatric developmental problems up to 3 years of age.? The results for neonatal and infant outcomes were reassuring and strengthened the overall idea that oncological treatment in pregnancy is feasible. However, the effect of antenatal chemotherapy on secondary malignant diseases or fertility later in life is still not known. Antenatal exposure to cancer treatment, and especially chemotherapy, was associated with an increased proportion of small-for-gestational-age children in some studies, ${ }^{4,6,8,9}$ whereas findings from other studies did not show an association. ${ }^{2,10}$ Further, several studies have described increased frequency of preterm delivery in patients with cancer during pregnancy. ${ }^{2,48}$ Nevertheless, these studies were small and could not identify which patients with cancer in pregnancy were at risk for negative obstetric or neonatal outcomes.

\section{Added value of this study}

To our knowledge, this study represents the largest cohort to describe detailed information on both clinical management and obstetric and neonatal outcomes in pregnant patients with cancer. We found that antenatal chemotherapy might increase the risk of neonatal complications. For small for gestational age, mainly platinum-based chemotherapy agents appeared associated. We also report changes in clinical management and obstetric and neonatal outcome over time. Over the past 20 years, more patients seem to be treated with chemotherapy during pregnancy, resulting in more livebirths and less prematurity than at the beginning of the study period.

\section{Implications of all available evidence}

Our findings are indicative of an increased knowledge and awareness about cancer treatment during pregnancy. Reduced prematurity has been shown to improve neonatal and longterm paediatric outcome; however, the use of chemotherapy during pregnancy might increase the likelihood of neonatal complications such as small for gestational age and NICU admission. Long-term paediatric outcomes need to be assessed in long-term follow-up studies. The risk factors from our study (eg, chemotherapy) should be used to identify pregnant patients with cancer who are at high obstetric risk and to refer them to academic hospitals for close surveillance in a multidisciplinary setting, and where specialised nursing care, physiotherapy, psychological guidance, and breastfeeding information additionally contribute to an optimal approach.

The aim of this study was to describe the oncological, obstetric, and neonatal data of the INCIP registry and to investigate changes in obstetric management and neonatal outcome over the past 20 years. We postulated that, over the years, patients would be more likely to receive treatment during pregnancy, which might have affected obstetric and neonatal outcomes. Further, we investigated whether type of cancer and treatment modality were related to adverse obstetric or neonatal outcomes. We hypothesised that chemotherapy during pregnancy might have resulted in increased adverse outcomes and were particularly interested in preterm, prelabour rupture of membranes (PPROM) or preterm contractions, small for gestational age, and admission to the neonatal intensive care unit (NICU), because these outcomes are common (appendix p 3).

\section{Methods}

\section{Study design and participants}

This study was a descriptive cohort study involving data from pregnant patients with cancer registered by all 37 centres (16 countries) participating in the INCIP registry (appendix p 7, 31). The INCIP was established in 2005 to investigate oncological care and obstetric, maternal, and neonatal outcomes in women with cancer 
during pregnancy. The aim was to register consecutive patients both retrospectively and prospectively. Before 2005, all patients were included retrospectively; after 2005, patients were included prospectively from the date that participating centres joined the INCIP registry. To identify patients for retrospective inclusion, hospitals used patient databases to identify all eligible patients within their hospital. Patient data were registered after written informed consent was obtained. This study was approved by the Ethical Committee of University Hospital Leuven (Belgian number B322201421061) and approval was obtained from national or institutional review boards or ethics committees at all participating centres.

\section{Procedures}

Patients diagnosed between Jan 1, 1996, and Nov 1, 2016, with primary invasive cancer and borderline ovarian cancer during pregnancy were eligible for participation. Patients with preinvasive disease or post-partum diagnosis were excluded, and we also excluded patients if we could not determine whether they matched the inclusion criteria because of missing data (eg, date of diagnosis or obstetrical dates). Detailed oncological, obstetric, and neonatal data were collected. Diagnosis was made according to local standards, but all included histopathological confirmation. We divided our cohort into three subgroups according to year of diagnosis: 1996-2004 (group 1), 2005-09 (group 2), and 2010 to November, 2016 (group 3). The differentiation between groups 1 and 2 was based on the start of our online registration study in 2005, after which most registrations were prospective. The differentiation between groups 2 and 3 was based on the publication date of the first INCIP report. ${ }^{4}$

TNM or FIGO stage IV disease and leukaemia were defined as systemic disease, whereas non-systemic disease was defined as TNM or FIGO stage I-III and all brain cancers. For surgery during pregnancy, we included only therapeutic or palliative surgical procedures that aimed to improve the maternal condition, not those for diagnostic procedures. PPROM was assessed following local protocols and was defined as preterm rupture of membranes without contractions. We defined perinatal mortality according to WHO guidelines ${ }^{11}$ as the number of stillbirths and deaths in the first week after birth. Major and minor congenital malformations were defined according to Eurocat. Birthweight percentiles were calculated according to the percentile calculator from the Gestation Network (v6.7.5.7[NL], 2014). The included parameters are shown in the appendix ( $\mathrm{p} 4)$. Birthweight less than the 10th percentile was deemed small for gestational age.

\section{Statistical analysis}

No dedicated sample size calculation was done for this descriptive study. We agreed on an analysis strategy beforehand, did not adapt this strategy based on obtained results, and fully report all results. We provide descriptive statistics of oncological, obstetric, and neonatal information. We analysed the relationship between cancer type, treatment modality, and obstetric and neonatal outcomes (PPROM or preterm contractions, small for gestational age, and NICU admission) with multiple logistic regression models using Firth bias correction. We stress that these models do not include a control group of patients without cancer, but compare patients with cancer during pregnancy with respect to the presence or absence of different characteristics or exposures.

For the obstetric outcome PPROM or preterm contractions, we based the regression analysis on the sample of singleton livebirths and stillbirths. For the two neonatal outcomes (small for gestational age and NICU admission), we based the analysis on the sample of singleton livebirths only. Both outcome variables and covariates in the models were prespecified. Key covariates in the models were cancer type, chemotherapy agent (alkylating agent excluding platinum, anthracyclines, antimetabolites, taxanes, platinum, or any other agent), and abdominal or cervical surgery. We added the following potential confounding variables without further data-driven variable selection: period of diagnosis, age at diagnosis, diagnosis in third pregnancy trimester, and systemic disease. We did not consider interaction terms. Alkylating chemotherapy agents were divided into platinum and other alkylating agents due to the relatively increased placental passage of carboplatin compared with other agents in baboon models, and the high placental passage of cisplatin in human beings. ${ }^{12,13} \mathrm{We}$ report adjusted odds ratios (ORs) with $95 \%$ CIs from the multiple logistic regression models. We report $\mathrm{p}$ values to measure the strength of the evidence against the null hypothesis of no relationship, but do not specify an $\alpha$ level and hence do not determine statistical significance. For the multiple regression models, we handled missing values for covariates or outcomes using multiple imputation (appendix p 5). ${ }^{14}$ As a sensitivity analysis, we compared results based on imputed data with results based on complete case analysis.

For the descriptive analysis and assessment of changes over 20 years in categorical patient characteristics, outcomes, and treatment modalities, we used univariable log-binomial regression models with year of diagnosis as a continuous predictor. We express results using relative risks $(\mathrm{RR})$ to describe the average change every 5 calendar years (appendix p 6) together with 95\% CIs. For continuous parameters, we used univariable linear regression with year of diagnosis as a continuous predictor, with results expressed as average change every 5 calendar years. Statistical significance was not determined. For this analysis, we did not impute missing data but rather used available cases. This analysis was prespecified, and was completed and reported for all parameters of interest.

The analysis was done with R 3.3.1.

This study is registered as an international observational cohort study with ClinicalTrials.gov, number NCT00330447.
For Eurocat see http://www eurocat-network.eu

For the Gestation Network calculator see http://www. gestation.net 


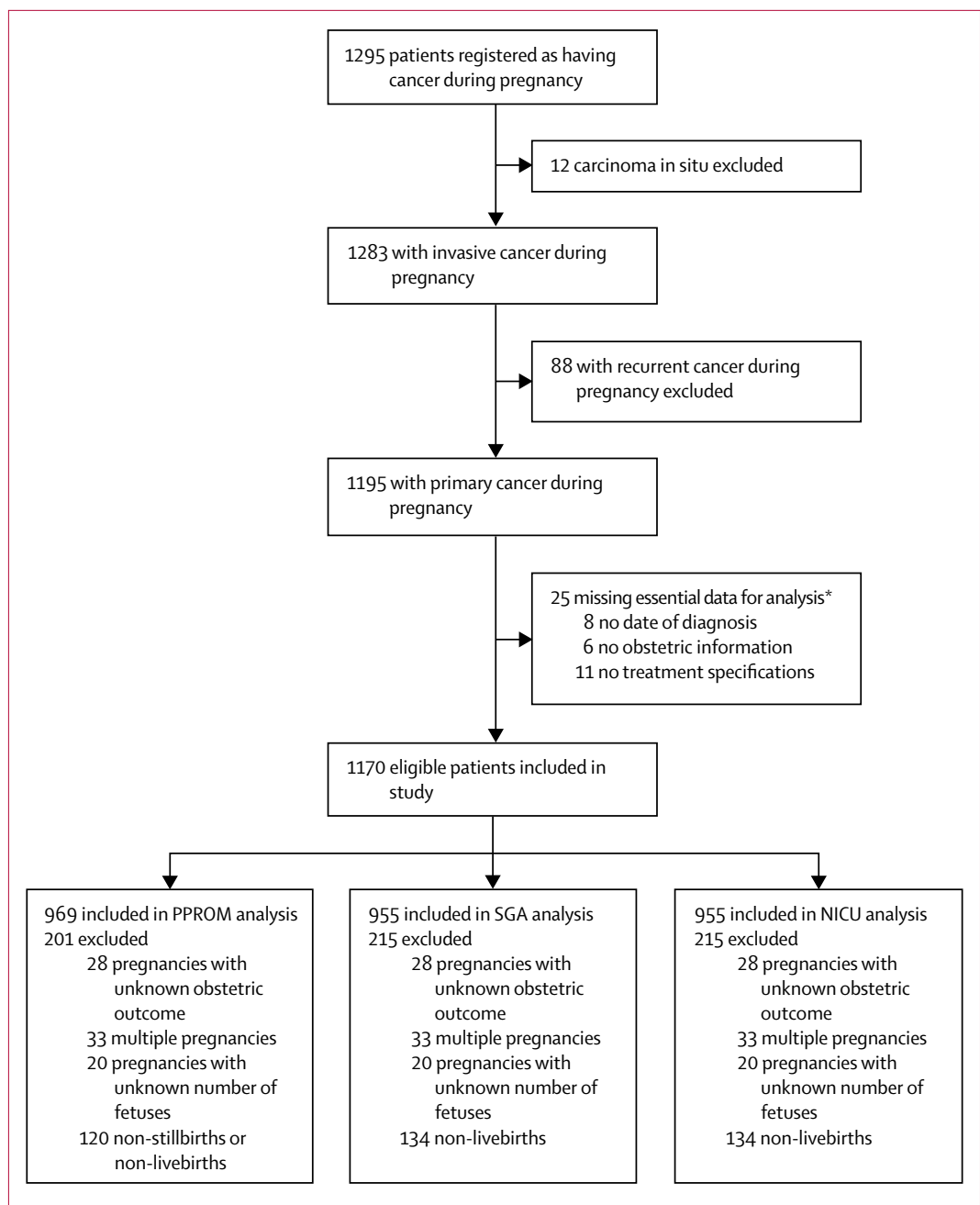

Figure 1: Study scheme

PPROM=preterm, prelabour rupture of membranes. $S G A=$ small for gestational age. $\mathrm{NICU}=$ neonatal intensive care unit. *Missing data essential to determine whether inclusion criteria are met.

For the study protocol see http://www.cancerinpregnancy. org/study-protocols
The full study protocol is available online; this manuscript is based on study part I and the primary objective of this study lies within the wider primary objective of the study protocol.

\section{Role of the funding source}

The study funders had no role in the study design, data collection, data analysis, interpretation of the data, or writing of the report. The corresponding author had full access to all the data in the study and had final responsibility for the decision to submit for publication.

\section{Results}

1170 consecutive patients were eligible from 37 centres in 16 countries (figure 1). Belgium had the highest accrual of patients (319 [27\%]), followed by the Netherlands (278 [24\%]), Italy (179 [15\%]), Russia (135 [12\%]), and the Czech Republic (100 [9\%]). Specifications of inclusion and

\begin{tabular}{|c|c|}
\hline & All patients $(n=1170)$ \\
\hline \multicolumn{2}{|l|}{ Age at diagnosis, years } \\
\hline Median (IQR; range) & $32(29-36 ; 16-53)$ \\
\hline Missing data & $13(1 \%)$ \\
\hline \multicolumn{2}{|l|}{ Period of diagnosis } \\
\hline 1996-2004 & $257(22 \%)$ \\
\hline 2005-09 & $376(32 \%)$ \\
\hline 2010-16 & $537(46 \%)$ \\
\hline \multicolumn{2}{|l|}{ Trimester of diagnosis* } \\
\hline Pregnant during treatment & 76/1098 (7\%) \\
\hline First trimester & 266/1098 (24\%) \\
\hline Second trimester & 490/1098 (45\%) \\
\hline Third trimester & 266/1098 (24\%) \\
\hline Missing data & $72(6 \%)$ \\
\hline \multicolumn{2}{|l|}{ Parity at diagnosis } \\
\hline Nulliparous & 486/1111 (44\%) \\
\hline Multiparous & $625 / 1111(56 \%)$ \\
\hline Missing data & $59(5 \%)$ \\
\hline \multicolumn{2}{|l|}{ Stage of disease } \\
\hline Local or regional & 893/1125 (79\%) \\
\hline Systemic† & 232/1125 (21\%) \\
\hline Missing data & $45(4 \%)$ \\
\hline \multicolumn{2}{|l|}{ Treatment received during pregnancy $\ddagger$} \\
\hline No treatment during pregnancy & $391(33 \%)$ \\
\hline Surgery & $454(39 \%)$ \\
\hline Abdominal or cervical surgery & $149(33 \%)$ \\
\hline Chemotherapy§ & $429(37 \%)$ \\
\hline Anthracyclines & $328(78 \%)$ \\
\hline Alkylating agents (excluding platinum) & $292(69 \%)$ \\
\hline Antimetabolites & $108(26 \%)$ \\
\hline Taxanes & $84(20 \%)$ \\
\hline Platinum & $74(17 \%)$ \\
\hline Other & $97(23 \%)$ \\
\hline Missing data & $6(1 \%)$ \\
\hline Radiotherapy & $29(2 \%)$ \\
\hline Targeted therapy & $33(3 \%)$ \\
\hline Other therapy & $52(4 \%)$ \\
\hline \multicolumn{2}{|c|}{ All singleton livebirths and stillbirths ( $n=969$ ) } \\
\hline \multicolumn{2}{|l|}{ Adverse obstetric outcome } \\
\hline PPROM or preterm contractions & $98(10 \%)$ \\
\hline \multicolumn{2}{|l|}{ All singleton livebirths $(n=955)$} \\
\hline \multicolumn{2}{|l|}{ Adverse neonatal outcome } \\
\hline Small for gestational age & 167/796 (21\%) \\
\hline Neonatal intensive care unit admission & 298/720 (41\%) \\
\hline \multicolumn{2}{|c|}{$\begin{array}{l}\text { Data are median (IQR; range) or } n \text { (\%). PPROM=preterm, prelabour rupture of } \\
\text { membranes. *Stratification per malignancy group is in the appendix ( } \mathrm{p} 11) \text {. } \\
\text { tSystemic disease was defined as TNM or FIGO stage IV disease and leukaemia; } \\
\text { non-systemic disease was defined as TNM or FIGO stage I-III and all brain cancers. } \\
\text { fPatients with multiple treatment modalities during pregnancy are placed in all } \\
\text { applicable groups, hence percentages add up to more than } 100 \text {. Stratification per } \\
\text { malignancy group of the different treatment combinations given during pregnancy } \\
\text { is in the appendix ( } \mathrm{p} 12) \text {. SCombination regimens consisting of more than one } \\
\text { chemotherapeutic agent were registered in } 351 \text { (83\%) of } 423 \text { patients with known } \\
\text { chemotherapy regimens. }\end{array}$} \\
\hline
\end{tabular}


an overview of missing values are given in the appendix (pp 8-10).

Baseline characteristics are shown in table 1, with distribution of cancer types and stage of disease shown in figure 2. Characteristics by cancer subtypes are in the appendix (p 11).

Of 1170 patients, 779 (67\%) received treatment during pregnancy, of which 574 (74\%) received a single treatment modality and 205 (26\%) a combination of different treatment modalities (table 2, appendix p 12). Combination regimens consisting of more than one chemotherapy agent were registered in 351 (83\%) of the 423 patients who received chemotherapy and who had known regimens. Abdominal or cervical surgery was done in 149 patients; 98 (69\%) of 143 of these patients had stage I disease, and for 104 (70\%) of 149 patients surgery was the only treatment modality used during pregnancy.

Of 1142 pregnancies with known obstetric outcome, there were $32(3 \%)$ twin pregnancies, one $(<1 \%)$ triplet pregnancy, and in $20(2 \%)$ pregnancies, the number of fetuses was unknown because of early miscarriage or termination. For the singleton pregnancies $(n=1089)$, $20(2 \%)$ ended in a miscarriage and $95(9 \%)$ were terminated. 55 (63\%) of 87 terminations were done in the first trimester, and 32 (37\%) were done in the second trimester; for eight patients $(1 \%)$, gestational age at termination was unknown. The main reasons for termination were start of oncological treatment or poor maternal prognosis (73 [77\%]), unwanted pregnancy (10 [11\%]), and fetal anomalies (4 [4\%]). The number of terminations per period of diagnosis and cancer type is shown in the appendix ( $\mathrm{p} 13)$. Five $(<1 \%)$ patients died during pregnancy (appendix p 14). Obstetric outcomes by cancer type for singleton livebirths are shown in table 3.

Of the 969 ongoing singleton pregnancies, seven (1\%) intrauterine fetal deaths and seven $(1 \%)$ perinatal deaths were reported (appendix $\mathrm{p}$ 14). PPROM or preterm contractions (98 [10\%] of 969 cases) were the most frequently reported obstetric complication among ongoing singleton pregnancies (appendix p 15). The remaining 955 (99\%) singleton pregnancies ended in a livebirth. Half of deliveries were preterm (430 [48\%] of 887 cases, excluding 67 with missing gestational age at birth). 373 (88\%) of 425 preterm deliveries for which data were available were iatrogenic, and $52(12 \%)$ were spontaneous. Data on all neonatal outcomes stratified by different variables are shown in the appendix (pp 16-17). Birthweight percentiles were calculated in 796 (83\%) of 955 singleton livebirths for which birthweight and gestational age at delivery were known (appendix p 18). 167 (21\%) of 796 children were small for gestational age. Information on NICU admission was available for 720 (75\%) of 955 children, and 298 (41\%) children were admitted. NICU admission was mainly related to prematurity (249 [84\%] of 298). Congenital malformations were reported in 32 (4\%) of

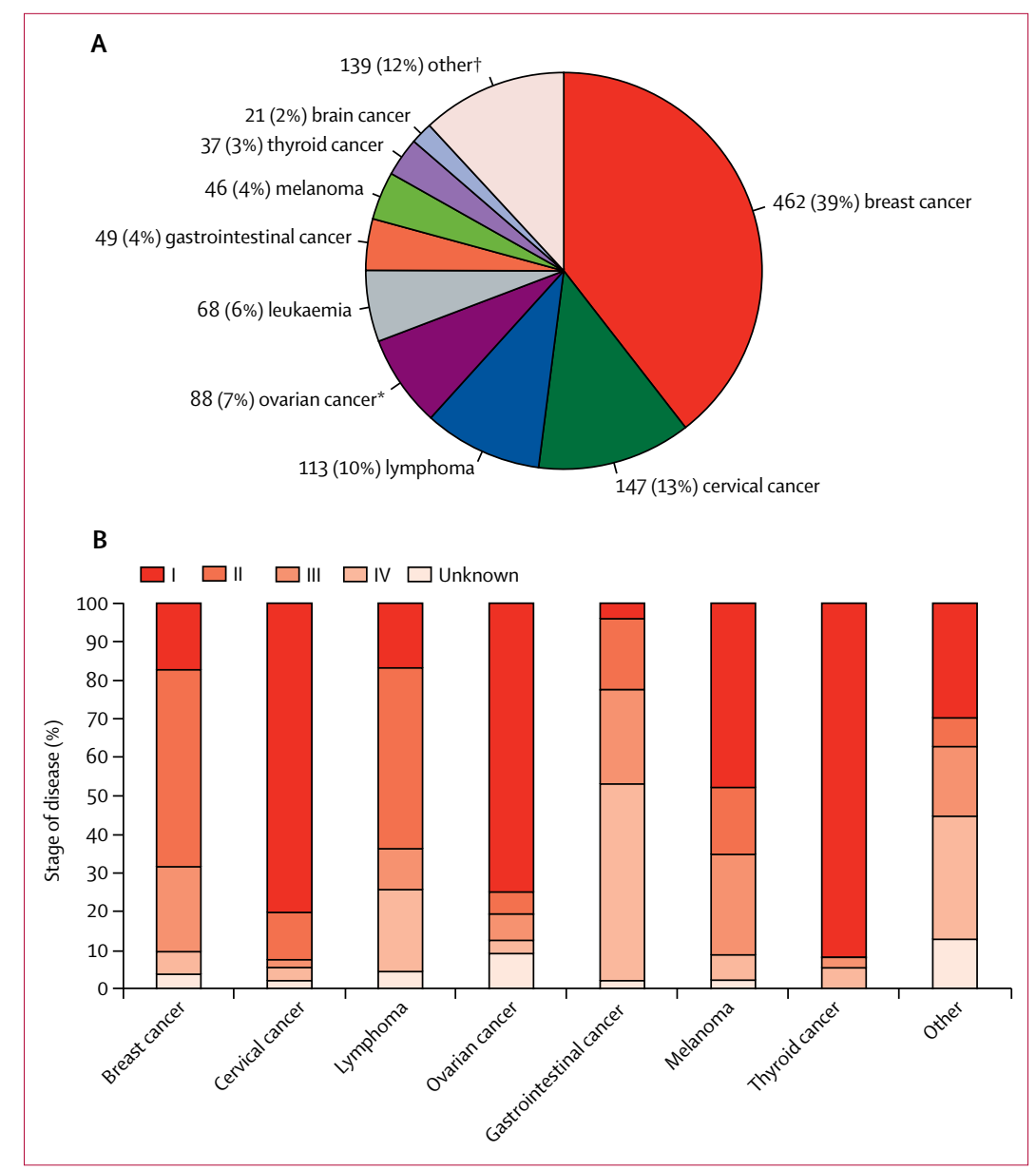

Figure 2: Distribution of cancers during pregnancy $(A)$ and stage of disease at diagnosis by cancer type (B) Stage of disease was available for all solid cancers with TNM or FIGO classification. *Ovarian cancers include borderline ovarian tumours. †Consists of 25 different cancer types.

\begin{tabular}{|c|c|c|c|c|c|c|c|}
\hline & Total & $\begin{array}{l}\text { No } \\
\text { treatment }\end{array}$ & $\begin{array}{l}\text { Any } \\
\text { surgery }\end{array}$ & Chemotherapy & Radiotherapy & $\begin{array}{l}\text { Targeted } \\
\text { and } \\
\text { hormonal } \\
\text { therapy* }\end{array}$ & $\begin{array}{l}\text { Other } \\
\text { therapy } \dagger\end{array}$ \\
\hline Breast & 462 & $116(25 \%)$ & 225 (49\%) & $248(54 \%)$ & $12(3 \%)$ & $7(2 \%)$ & 0 \\
\hline Cervix & 147 & $83(56 \%)$ & $32(22 \%)$ & $37(25 \%)$ & $2(1 \%)$ & 0 & 0 \\
\hline Lymphoma & 113 & $41(36 \%)$ & $8(7 \%)$ & $66(58 \%)$ & $4(4 \%)$ & $18(16 \%)$ & 0 \\
\hline Ovarian & 88 & $23(26 \%)$ & $64(73 \%)$ & $21(24 \%)$ & 0 & 0 & 0 \\
\hline Leukaemia & 68 & $22(32 \%)$ & 0 & $23(34 \%)$ & $1(1 \%)$ & $7(10 \%)$ & $15(22 \%)$ \\
\hline Gastrointestinal & 49 & $19(39 \%)$ & $21(43 \%)$ & $16(33 \%)$ & 0 & 0 & 0 \\
\hline Melanoma & 46 & $12(26 \%)$ & $33(72 \%)$ & 0 & $2(4 \%)$ & 0 & 0 \\
\hline Thyroid & 37 & $7(19 \%)$ & $30(81 \%)$ & 0 & $1(3 \%)$ & 0 & 0 \\
\hline Brain & 21 & $11(52 \%)$ & $10(48 \%)$ & $1(5 \%)$ & $1(5 \%)$ & 0 & 0 \\
\hline Other & 139 & $57(41 \%)$ & $31(22 \%)$ & $17(12 \%)$ & $6(4 \%)$ & $1(1 \%)$ & $37(27 \%)$ \\
\hline Total & 1170 & $391(33 \%)$ & $454(39 \%)$ & $429(37 \%)$ & $29(2 \%)$ & $33(3 \%)$ & $52(4 \%)$ \\
\hline \multicolumn{8}{|c|}{$\begin{array}{l}\text { Table shows data for all } 1170 \text { patients. Patients with multiple treatment modalities during pregnancy are placed in all } \\
\text { applicable groups. *Targeted and hormonal therapy included rituximab (18 patients), imatinib (seven patients), } \\
\text { trastuzumab (three patients), tamoxifen (three patients), lorlatinib (one patient), and trastuzumab plus pertuzumab } \\
\text { (one patient). †Other therapy included interferon ( } 52 \text { patients). }\end{array}$} \\
\hline
\end{tabular}




\begin{tabular}{|c|c|c|c|c|c|c|c|c|}
\hline & Total & Miscarriage & $\begin{array}{l}\text { Termination of } \\
\text { pregnancy }\end{array}$ & Stillbirth* & $\begin{array}{l}\text { Livebirth } \\
<37 \text { weeks }\end{array}$ & $\begin{array}{l}\text { Livebirth } \\
\geq 37 \text { weeks }\end{array}$ & $\begin{array}{l}\text { Livebirth } \\
\text { gestational age } \\
\text { unknown }\end{array}$ & $\begin{array}{l}\text { Maternal death } \\
\text { during pregnancy }\end{array}$ \\
\hline Breast cancer & 428 & $6(1 \%)$ & $26(6 \%)$ & $1(<1 \%)$ & $184(43 \%)$ & $182(43 \%)$ & $28(7 \%)$ & $1(<1 \%)$ \\
\hline Cervical cancer & 140 & $2(1 \%)$ & $21(15 \%)$ & $2(1 \%)$ & $72(51 \%)$ & $37(26 \%)$ & $6(4 \%)$ & 0 \\
\hline Lymphoma & 107 & 0 & $8(7 \%)$ & $3(3 \%)$ & $48(45 \%)$ & 45 (42\%) & $3(3 \%)$ & 0 \\
\hline Ovarian cancer & 83 & $3(4 \%)$ & $3(4 \%)$ & 0 & $21(25 \%)$ & $53(64 \%)$ & $3(4 \%)$ & 0 \\
\hline Leukaemia & 64 & $5(8 \%)$ & $6(9 \%)$ & $2(3 \%)$ & $26(41 \%)$ & $25(39 \%)$ & 0 & 0 \\
\hline $\begin{array}{l}\text { Gastrointestinal } \\
\text { cancer }\end{array}$ & 47 & $2(4 \%)$ & $4(9 \%)$ & $2(4 \%)$ & $29(62 \%)$ & $8(17 \%)$ & $1(2 \%)$ & $1(2 \%)$ \\
\hline Melanoma & 43 & 0 & $2(5 \%)$ & 0 & $3(7 \%)$ & $34(79 \%)$ & $3(7 \%)$ & $1(2 \%)$ \\
\hline Thyroid cancer & 37 & 0 & $4(11 \%)$ & 0 & $1(3 \%)$ & $32(86 \%)$ & 0 & 0 \\
\hline Brain cancer & 19 & 0 & $2(11 \%)$ & 0 & $9(47 \%)$ & $6(32 \%)$ & 0 & $2(11 \%)$ \\
\hline $\begin{array}{l}\text { Other malignant } \\
\text { diseases }\end{array}$ & 121 & $2(2 \%)$ & $19(16 \%)$ & $4(3 \%)$ & $37(31 \%)$ & $36(30 \%)$ & $23(19 \%)$ & 0 \\
\hline Total & 1089 & $20(2 \%)$ & $95(9 \%)$ & $14(1 \%)$ & $430(39 \%)$ & $458(42 \%)$ & $67(6 \%)$ & $5(<1 \%)$ \\
\hline
\end{tabular}

\begin{tabular}{|c|c|c|c|c|c|c|}
\hline & \multicolumn{2}{|c|}{ PPROM or preterm contractions } & \multicolumn{2}{|c|}{ Small for gestational age } & \multicolumn{2}{|c|}{ Neonatal intensive care unit admission } \\
\hline & OR $(95 \% \mathrm{Cl})$ & $\mathrm{p}$ value & OR $(95 \% \mathrm{Cl})$ & $p$ value & OR $(95 \% \mathrm{Cl})$ & pvalue \\
\hline Cancer type & .. & 0.16 &.. & 0.86 &.. & $<0.0001$ \\
\hline Breast cancer* & Reference &.. & Reference &.. & Reference &.. \\
\hline Cervical cancer & $0.74(0.27-2.04)$ &.. & $0.75(0.36-1.55)$ &.. & $2 \cdot 22(1 \cdot 19-4 \cdot 15)$ &.. \\
\hline Lymphoma & $1.24(0.49-3 \cdot 12)$ &.. & $1.17(0.52-2.60)$ & .. & $1.04(0.53-2.04)$ & .. \\
\hline Ovarian cancer & $0.60(0.16-2.30)$ &.. & $0.39(0.14-1.09)$ &.. & $0.60(0.26-1.38)$ &.. \\
\hline Leukaemia & $2.45(0.80-7 \cdot 48)$ &.. & $0.68(0.23-2.03)$ &.. & $1.27(0.53-3.03)$ &.. \\
\hline Gastrointestinal cancer & $0.33(0.06-1.96)$ &.. & $0.80(0.29-2.22)$ &.. & $7 \cdot 13(2 \cdot 86-17 \cdot 7)$ &.. \\
\hline Melanoma & $0.76(0.19-3.12)$ &.. & $0.90(0.29-2.76)$ &.. & $0.36(0.13-1.04)$ &.. \\
\hline Thyroid cancer & $0.52(0.09-3.12)$ &.. & $0.73(0.21-2.58)$ &.. & $0.14(0.02-0.90)$ &.. \\
\hline Other malignant diseases & $0.44(0.15-1 \cdot 31)$ & $\cdot \cdot$ & $0.82(0.36-1.83)$ & .. & $1.42(0.73-2 \cdot 75)$ & .. \\
\hline Period of diagnosis & .. & 0.69 &.. & 0.32 &.$\cdot$ & 0.019 \\
\hline 1996-2004 & Reference &.$\cdot$ & Reference & $\cdot$. & Reference & .. \\
\hline $2005-09$ & $0.81(0.44-1 \cdot 48)$ & $\cdot$. & $0 \cdot 77(0 \cdot 45-1 \cdot 31)$ &.. & $0.73(0.48-1 \cdot 11)$ &. \\
\hline 2010-16 & $0.77(0.43-1 \cdot 39)$ &.. & $1.04(0.63-1.73)$ &.. & $0.55(0.36-0.84)$ &.. \\
\hline Age at diagnosis (per 5 years) & $1.08(0.86-1.35)$ & 0.53 & $1 \cdot 36(1 \cdot 11-1 \cdot 68)$ & 0.0033 & $0.98(0.82-1.17)$ & 0.65 \\
\hline Diagnosis in third trimester vs before & $0.64(0.35-1.15)$ & 0.14 & $0.78(0.48-1 \cdot 27)$ & $0 \cdot 33$ & $1.13(0.77-1 \cdot 65)$ & 0.52 \\
\hline Systemic vs non-systemic disease & $1.43(0 \cdot 70-2 \cdot 92)$ & 0.34 & $1.86(1.04-3 \cdot 33)$ & 0.039 & $1.14(0.68-1.93)$ & 0.52 \\
\hline Chemotherapeutic agents &.. & 0.056 &.. & $<0.0001$ &.. & 0.0086 \\
\hline Non-platinum alkylating (yes vs no) & $2 \cdot 02(0.81-5 \cdot 02)$ &.. & $2.08(0.88-4.91)$ & .. & $0.88(0.46-1 \cdot 70)$ &.$\cdot$ \\
\hline Anthracyclines (yes vs no) & $1.11(0.42-2 \cdot 92)$ &.. & $0.50(0.21-1 \cdot 22)$ &.. & $1.21(0.62-2 \cdot 38)$ &. \\
\hline Antimetabolites (yes vs no) & $0.89(0.46-1.71)$ &.. & $1 \cdot 24(0 \cdot 70-2 \cdot 22)$ &.. & $1.03(0.60-1.74)$ &.. \\
\hline Taxanes (yes vs no) & $1.11(0.53-2 \cdot 33)$ &.. & $2 \cdot 07(1.11-3 \cdot 86)$ &.. & $2 \cdot 37(1 \cdot 31-4 \cdot 28)$ &.. \\
\hline Platinum (yes vs no) & $2 \cdot 29(0.79-6.63)$ &.. & $3 \cdot 12(1.45-6 \cdot 70)$ &.. & $1.66(0.77-3 \cdot 55)$ &. \\
\hline Other (yes vs no) & $1.48(0.61-3.63)$ &.. & $2 \cdot 34(1.04-5 \cdot 25)$ &.. & $1.63(0.78-3.38)$ &.. \\
\hline Abdominal or cervical surgery (yes vs no) & $0.42(0.15-1.16)$ & 0.083 & $1 \cdot 31(0.67-2 \cdot 59)$ & 0.45 & $0.30(0.17-0.55)$ & $<0.0001$ \\
\hline \multicolumn{7}{|c|}{ 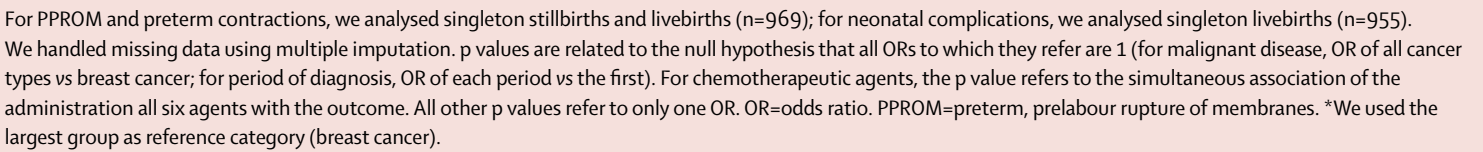 } \\
\hline
\end{tabular}


721 liveborn singletons with data available, split between minor (17 [2\%]) and major (15 [2\%]) malformations. Three pregnancies were terminated because of fetal anomalies (hydrocephalus, trisomy 21, and unspecified major malformations). Anomalies did not differ between the different treatment modalities (appendix p 19).

Results from the multiple logistic regression models, including associations for the prespecified potential confounders, are shown in table 4; model coefficients and standard errors are in the appendix (p 20). The multiple regression model for small for gestational age provided support for a relationship between chemotherapy and this outcome, particularly for platinum-based chemotherapy (OR 3.12, 95\% CI 1.45-6.70). Other agents such as non-platinum alkylating chemotherapy or taxanes might also be related $(\mathrm{OR}>2)$, but lower confidence limits were low. No meaningful relationships were observed between cancer type and abdominal or cervical surgery (table 4, appendix p 21).

For NICU admission, there seemed to be a strong, independent association with cancer type; compared with breast cancer, gastrointestinal cancer had the highest association with NICU admission (OR 7.13, 95\% CI 2.86-17.7), whereas thyroid cancer had the lowest $(0 \cdot 14,0 \cdot 02-0 \cdot 90$; appendix p 22). There was also support for an association between chemotherapy and NICU admission (table 4). Finally, the data suggested that abdominal or cervical surgery was related to reduced frequency of NICU admission (table 4).

For PPROM or preterm contractions, the least common of the three investigated complications (only 98 registered instances), results were largely inconclusive for all variables because of high standard errors (table 4; appendix pp 20, 23). The relationship between chemotherapy and PPROM or preterm contractions was in line with our hypothesis, with ORs greater than 2 for platinum and non-platinum alkylating agents.

A sensitivity analysis of all three outcomes based on complete cases provided similar results (appendix p 24).

Every 5 years, the likelihood of patients receiving treatment during pregnancy increased (RR 1·10, 95\% CI $1 \cdot 05-1 \cdot 15)$, mainly related to an increase of chemotherapeutic treatment $(1 \cdot 31,1 \cdot 20-1.43$; figure $3 \mathrm{~A}$; appendix $\mathrm{p}$ 25). There was little change in the likelihood of receiving surgery $(0 \cdot 99,0 \cdot 92-1 \cdot 07)$. Radiotherapy became less frequent and targeted therapy more frequent, but these modalities were uncommon in general. Every 5 years, we observed an increase of $2 \cdot 6$ days $(95 \% \mathrm{CI}-1 \cdot 1$ to $6 \cdot 3$ ) in the gestational age of the last chemotherapy cycle given during pregnancy.

Every 5 years there was, on average, an increased likelihood of livebirth among singleton pregnancies (RR 1.04, 95\% CI 1.01-1.06), a reduction in the risk of preterm livebirth $(0 \cdot 93,0 \cdot 86-0.99)$, and a reduction in the risk of iatrogenic preterm livebirth $(0 \cdot 91,0 \cdot 84-0 \cdot 98$; figure 3B). In line with the decrease in preterm deliveries, NICU admissions decreased (RR $0.91,95 \%$ CI
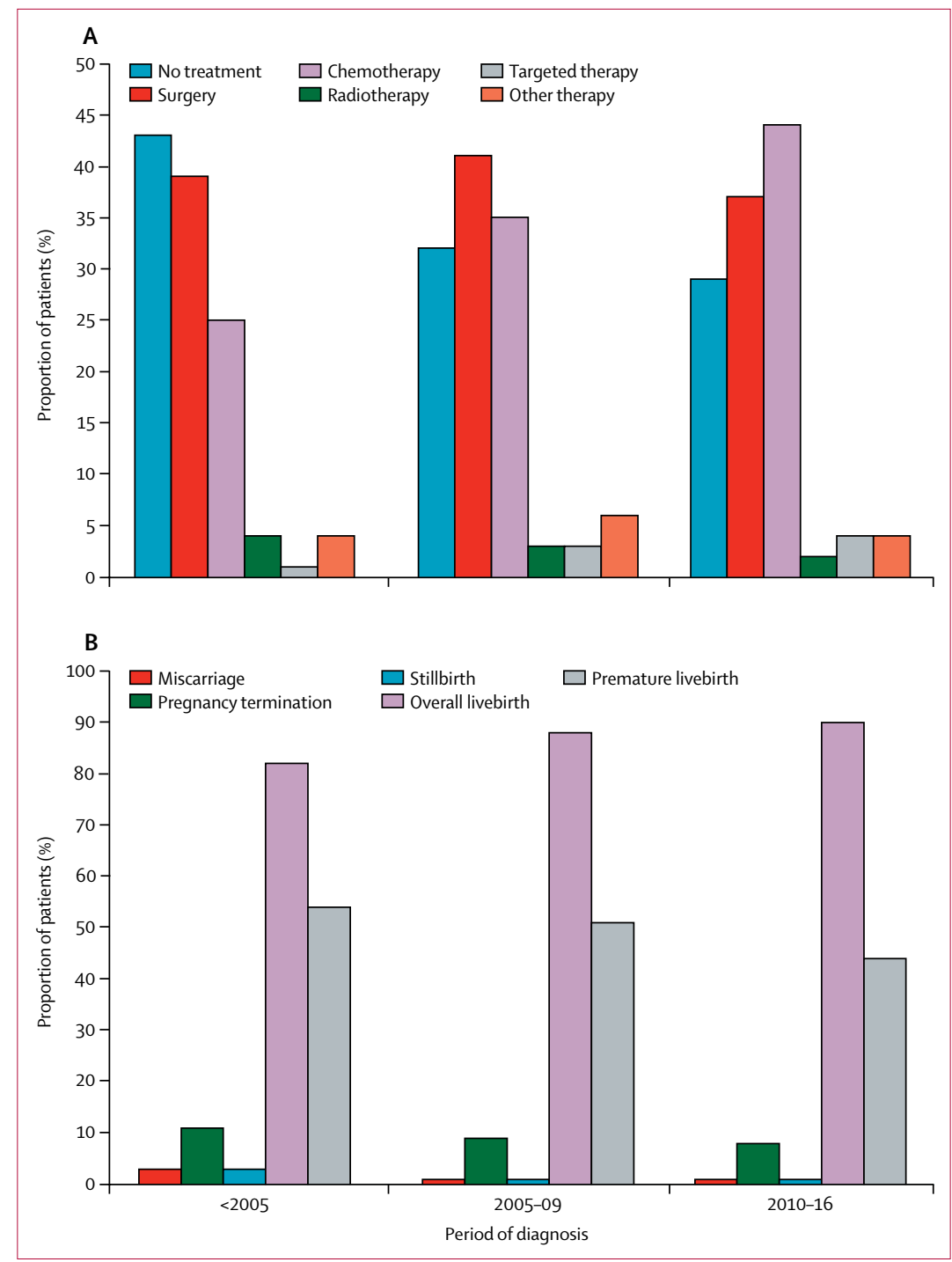

Figure 3: Changes in oncological management (A) and obstetric outcome (B) over 20 years Management changes are shown for all 1170 patients; obstetric outcome is shown for all 1089 singleton pregnancies with known obstetric outcome.

$0 \cdot 83-0 \cdot 99)$ and PPROM or preterm contractions decreased $(0 \cdot 97,0 \cdot 80-1 \cdot 18)$ every 5 years, whereas the risk of small for gestational age increased $(1 \cdot 16$, $0 \cdot 99-1 \cdot 35)$.

\section{Discussion}

In this cohort study of obstetric and neonatal outcomes for patients with cancer diagnosed during pregnancy, our data suggested a relationship between platinum-based chemotherapy and small for gestational age, and between taxane chemotherapy and NICU admission. Overall, the likelihood of NICU admission seemed to depend on cancer type. Unexpectedly, the data suggested that abdominal or cervical surgery was related to a lower frequency of NICU admission. Other associations 
between treatment and cancer type were less clear. The frequency of malformations was low (4\%), and equivalent to that in the general population (frequency of major malformations of $2 \%$ in our study vs $2 \cdot 5-3 \%$ in the general population $\left.{ }^{15}\right)$. Over the 20 -year period of the study, the proportion of pregnancies ending in a livebirth increased, and every 5 years the likelihood of chemotherapeutic treatment during pregnancy increased. In line with the increasing use of chemotherapy, the frequency of small for gestational age also rose for each 5 -year period of the study. These results strengthen the recommendation to involve hospitals with obstetric high-care units when treating pregnant patients with chemotherapy. The complexity of dealing with two patients at once highlights the need for a multidisciplinary approach.

The increased use of chemotherapy during pregnancy in combination with an increase in livebirths might be attributable to changing treatment regimens during the period of registration, in combination with reassuring results on antenatal chemotherapy exposure. Since 1996 , 25 cohort studies, each including more than 50 patients with cancer during pregnancy, have focused on obstetric outcomes (appendix pp 26-30). Findings from these studies showed a high frequency of preterm birth, but the relationship between small for gestational age and cancer treatment during pregnancy remained inconclusive. Investigators of several matched cohort studies have described reassuring fetal outcomes after chemotherapy during pregnancy, with no congenital, neurological, or psychological abnormalities detected in children exposed to antenatal chemotherapy., ${ }^{7,16}$ These data showing reassuring fetal, neonatal, and infant outcomes up to 3 years, together with maternal survival similar to that of non-pregnant women diagnosed with or treated for cancer, ${ }^{5}$ might have guided decision making and resulted in the increase in cancer treatment over time that we observed.

Our findings confirm results of a high overall frequency of premature birth (48\%) in patients with cancer during pregnancy, as shown by several previous cohort studies (appendix pp 26-30). Preterm birth increases the risk of perinatal morbidity and mortality, and neurodevelopmental impairment later in life, with a direct correlation between lower gestational age at delivery and negative outcomes. ${ }^{18}$ $\mathrm{Lu}$ and colleagues ${ }^{8}$ observed an increased risk of neonatal mortality in patients with cancer during pregnancy (incidence rate ratio $2 \cdot 7,95 \%$ CI $1 \cdot 3-5 \cdot 6$ ), which was caused by prematurity in $89 \%$ of cases. Our study showed inconclusive results for the association between antenatal chemotherapy exposure and PPROM or preterm contractions. As for other risk factors for spontaneous premature delivery, we hypothesised that patients undergoing abdominal or cervical surgery would be at increased risk of PPROM or preterm contractions, but our multiple regression analysis did not support such an effect. This finding could be explained by the high proportion of patients with stage I disease $(69 \%)$ or patients who received surgical therapy only $(70 \%)$ in this group, because these patients have no potential risk factors for adverse obstetric or neonatal outcomes. ${ }^{19,20} \mathrm{We}$ observed a decline in frequency of preterm birth during the study period every 5 calendar years, which was mainly attributed to the reduced frequency of iatrogenic premature delivery. This decline could be attributable to the tendency to continue chemotherapy for longer during pregnancy, allowing postponement of delivery for the benefit of the child. We realise that the effect (increase in gestational age of the last chemotherapy cycle of 2.6 days [95\% CI $-1 \cdot 1$ to $6 \cdot 3$ ] for each 5 -year study period) was not strong. This finding could be accounted for by the fact that reassuring results on antenatal chemotherapy were published only a few years ago; the follow-up in this cohort might not be long enough to fully evaluate this change.

The tendency to treat an increased number of patients with chemotherapy during pregnancy could also have adverse consequences. For example, we noted an increased frequency of small for gestational age. Preterm birth, perinatal morbidity, and perinatal mortality in the first weeks of life, and cardiovascular and metabolic diseases later in life, are more frequently seen in these children than in children of average weight at birth..$^{21,22}$ Several studies have highlighted an increased frequency of small for gestational age in children from patients with cancer during pregnancy (appendix p 26). Still, the influence of supportive medication, maternal stress, and malnutrition on the occurrence of small for gestational age cannot be excluded.

We hypothesised a relationship between systemic disease and small for gestational age, for which our analysis provided mild support. In these patients, nutritional state and other factors (including general condition, fatigue, and circulating cytokines) might be compromised compared with patients with localised malignant disease, irrespective of the treatment given.

Our findings further suggest a relationship between chemotherapy, mainly platinum-based agents, and small for gestational age, as hypothesised. Several factors might contribute to such a relationship. Chemotherapy agents have several toxic properties, and some cause direct damage to DNA or interfere with DNA replication (eg, alkylating agents, antimetabolites). This direct DNA damage might affect placental development and fetal blood supply. Additional indirect effects of chemotherapy (eg, induction of vasculopathy or inflammation), or the maternal illness itself (associated with malnutrition, anaemia, and high maternal stress), could further contribute to restricted fetal growth. ${ }^{23-25}$ Besides the effects of chemotherapy on fetal growth, higher maternal age has an additional effect.

Fortunately, the reduced birthweights in chemotherapy-exposed children tend to recover, with normal values for weight, height, and head circumference in the first months of childhood. ${ }^{6,7}$ 
In pregnant patients with cancer, it is important to recognise obstetric and neonatal risks associated with cancer and its treatment modalities. The tendency to avoid preterm deliveries by treating cancer during pregnancy needs to be balanced against an increased risk of small for gestational age. The short-term and longterm risks of this outcome are important to consider, but the risks associated with preterm birth are also of great importance. More long-term research comparing the risks in these two groups is necessary.

To our knowledge, this analysis includes the largest cohort of patients diagnosed with cancer in pregnancy reported to date. This study adds to the identification of patients at high risk for obstetric and neonatal complications. However, limitations to this study need to be addressed. First, data were missing for some patients, especially for neonatal outcomes. This problem can be attributed to the participating hospitals, some of which are specialised in either oncology or obstetrics and perinatology, leading to lack of either oncological or obstetric and neonatal information. Due to the necessity to report obstetric complications when observed, but no explicit mention of the absence of complications, it is possible that the occurrence of obstetric complications is under-reported. Second, we documented sampling data from our online database, which was registered on a voluntary basis by the participating INCIP centres and included retrospective cases; the incidence of different tumour types and treatment methods given during pregnancy might differ from pregnant patients with cancer treated at other centres or in other countries. Although all participating centres attempted to register all of their consecutive cases rigorously, some selection bias for retrospectively included cases cannot be excluded. Third, a common issue in observational studies is the presence of confounding, in our case between treatment and patient or tumour characteristics. Fourth, due to the rarity of cancer in pregnancy and the changes in cancer treatment over the past few years, we encountered small group sizes for some cancer types and treatment methods, for which subgroup analysis was not possible.

The observation of increased small for gestational age with chemotherapy exposure needs further research. In our study, percentiles were calculated at birth, and we do not know if there were specific decreases in fetal size from start of chemotherapy. However, the measurement of fetal weight percentiles accurately during pregnancy is difficult, because it is observer-dependent and there are no fetal charts available worldwide that include ethnic and gender differences and the effect of parental weight and height.

Further research on placental pathophysiology and the effect of specific chemotherapy agents, as well as more research into rare tumour types and infrequently used treatment modalities, are needed to provide all patients diagnosed with cancer during pregnancy with the best individualised management plan to optimise obstetric and neonatal outcomes. Participation in the INCIP registry is recommended to accomplish this goal.

To register for INCIP see http://

Contributors

FA designed the study. JdH, MV, KVC, and FA gathered, interpreted, and vouch for the data. JdH, MV, KVC, BVC, and FA analysed the data All other authors were involved in data gathering. The first draft of the Article was written by JdH, MV, KVC, CARL, and FA, and all other authors revised the Article for the final draft. All authors agreed with the submitted Article.

Declaration of interests

KVC reports grants from University Hospitals Leuven during the conduct of this study. The other authors declare no competing interests.

\section{Acknowledgments}

We thank all medical specialists, outpatient department managers, data and case managers, secretaries, and all other people who collected all data necessary for completing the database. This project is supported by the Research Foundation-Flanders (FWO) in Belgium (grant no G070514N) and the European Research Council (CRADLE consolidator grant, grant no ZKD0230) and by Charles University research project Progres Q28-Oncology, UNCE 204013, and by Ministry of Health of the Czech Republic, grant no 15-28933A. FA is senior clinical investigator and MV is a research fellow of the Research Foundation-Flanders (FWO).

\section{References}

1 Smith LH, Danielsen B, Allen ME, Cress R. Cancer associated with obstetric delivery: results of linkage with the California cancer registry. Am J Obstet Gynecol 2003; 189: 1128-35.

2 Lee YY, Roberts CL, Dobbins T, et al. Incidence and outcomes of pregnancy-associated cancer in Australia, 1994-2008: a population-based linkage study. BJOG 2012; 119: 1572-82.

3 Parazzini F, Franchi M, Tavani A, Negri E, Peccatori FA. Frequency of pregnancy related cancer: a population based linkage study in Lombardy, Italy. Int J Gynecol Cancer 2017; 27: 613-19.

4 Van Calsteren K, Heyns L, De Smet F, et al. Cancer during pregnancy: an analysis of 215 patients emphasizing the obstetrical and the neonatal outcomes. J Clin Oncol 2010; 28: 683-89.

5 Stensheim H, Moller B, van Dijk T, Fossa SD. Cause-specific survival for women diagnosed with cancer during pregnancy or lactation: a registry-based cohort study. J Clin Oncol 2009; 27: 45-51.

6 Amant F, Van Calsteren K, Halaska MJ, et al. Long-term cognitive and cardiac outcomes after prenatal exposure to chemotherapy in children aged 18 months or older: an observational study. Lancet Oncol 2012; 13: 256-64.

7 Amant F, Vandenbroucke T, Verheecke M, et al. Pediatric outcome after maternal cancer diagnosed during pregnancy. $N$ Engl J Med 2015; 373: 1824-34

8 Lu D, Ludvigsson JF, Smedby KE, et al. Maternal cancer during pregnancy and risks of stillbirth and infant mortality. J Clin Oncol 2017; 35: 1522-29.

9 Loibl S, Han SN, von Minckwitz G, et al. Treatment of breast cancer during pregnancy: an observational study. Lancet Oncol 2012; 13: 887-96.

10 Cardonick E, Usmani A, Ghaffar S. Perinatal outcomes of a pregnancy complicated by cancer, including neonatal follow-up after in utero exposure to chemotherapy: results of an international registry. Am J Clin Oncol 2010; 33: 221-28.

11 WHO. Maternal, newborn, child and adolescent health. http://www. who.int/maternal_child_adolescent/topics/maternal/maternal_ perinatal/en/ (accessed Jan 16, 2018).

12 Van Calsteren K, Verbesselt R, Devlieger R, et al. Transplacental transfer of paclitaxel, docetaxel, carboplatin, and trastuzumab in a baboon model. Int J Gynecol Cancer 2010; 20: 1456-64.

13 Köhler C, Oppelt P, Favero G, et al. How much platinum passes the placental barrier? Analysis of platinum applications in 21 patients with cervical cancer during pregnancy. Am J Obstet Gynecol 2015; 213: 206.e1-5.

14 White IR, Royston P, Wood AM. Multiple imputation using chained equations: Issues and guidance for practice. Stat Med 2011; 30: 377-99.

15 Dolk H, Loane M, Garne E. The prevalence of congenital anomalies in Europe. Adv Exp Med Biol 2010; 686: 349-64. 
16 Avilés A, Neri N. Hematological malignancies and pregnancy: a final report of 84 children who received chemotherapy in utero. Clin Lymphoma 2001; 2: 173-77.

17 Cardonick E, Gringlas M. Development of children born to mothers with cancer during pregnancy: comparing in utero chemotherapy-exposed children with nonexposed controls. Am J Obstet Gynecol 2015; 212: 658.e1-8.

18 Wang ML, Dorer DJ, Fleming MP, Catlin EA. Clinical outcomes of near-term infants. Pediatrics 2004; 114: 372-76.

19 Boucek J, de Haan J, Halaska MJ, et al. Maternal and obstetrical outcome in 35 cases of well-differentiated thyroid carcinoma during pregnancy. Laryngoscope 2017; published online Oct 8. DOI:10.1002/ lary.26936

20 de Haan J, Lok CA, de Groot CJ, et al, for the International Network on Cancer, Infertility and Pregnancy (INCIP). Melanoma during pregnancy: a report of 60 pregnancies complicated by melanoma. Melanoma Res 2017; 27: 218-23.
21 Pallotto EK, Kilbride HW. Perinatal outcome and later implications of intrauterine growth restriction. Clin Obstet Gynecol 2006; 49: 257-69.

22 Sankaran S, Kyle PM. Aetiology and pathogenesis of IUGR Best Pract Res Clin Obstet Gynaecol 2009; 23: 765-77.

23 Salafia CM. Placental pathology of fetal growth restriction. Clin Obstet Gynecol 1997; 40: 740-49.

24 Wadhwa PD, Garite TJ, Porto M, et al. Placental corticotropin-releasing hormone (CRH), spontaneous preterm birth, and fetal growth restriction: a prospective investigation. Am J Obstet Gynecol 2004; 191: 1063-69.

25 Nulman I, Laslo D, Fried S, Uleryk E, Lishner M, Koren G. Neurodevelopment of children exposed in utero to treatment of maternal malignancy. Br J Cancer 2001; 85: 1611-18. 